\title{
Anomalous processes at high temperature and density in a two-dimensional linear $\sigma$ model
}

\author{
François Gelis \\ Physics Department, Nuclear Theory, Brookhaven National Laboratory, Upton, New York 11973-5000
}

Michel H. G. Tytgat

CERN, Theory Division, CH-1211 Geneva 23, Switzerland

(Received 1 March 2000; published 27 November 2000)

\begin{abstract}
We use the two-dimensional $\sigma$ model as a toy model to study the behavior of anomalous amplitudes in the limit where the constituent quark mass is small. Symmetry arguments show that the $\pi^{0} \rightarrow \gamma$ amplitude should vanish if $m \rightarrow 0$, but we show that the one-loop $\pi^{0} \gamma$ amplitude does not satisfy this property because of infrared singularities. However, when a proper regularization (resummation of a thermal mass, for instance) is taken into account, this amplitude vanishes as expected. We also study the amplitude $\pi^{0} \sigma \rightarrow \gamma$ and show that it does not vanish in the same limit.
\end{abstract}

DOI: 10.1103/PhysRevD.63.016001

PACS number(s): 11.10.Wx

\section{INTRODUCTION}

In past years, many works have been devoted to the study of anomalous processes at finite temperature and density, and in particular near the chiral symmetry restoration. In particular, Pisarski $[1,2]$ concluded that the neutral pion decay amplitude $\pi^{0} \rightarrow 2 \gamma$ vanishes when chiral symmetry is restored. ${ }^{1}$ This conclusion is based on a direct calculation of the corresponding diagrams at finite temperature (and zero density) in the imaginary time formalism, and on symmetry considerations that forbid certain couplings in the symmetric phase. An additional conclusion was that this decay might be replaced by $\pi^{0} \sigma \rightarrow 2 \gamma$ in the chirally symmetric phase (which is allowed by the same symmetry argument), as indicated by a calculation of the box diagram at finite temperature.

This result has been confirmed by Baier, Dirks and Kober [11] and by Salcedo [12] using functional approaches in which one integrates out the fermions at the level of the generating functional. Salcedo in [12] gives also general arguments according to which $\pi^{0} \rightarrow 2 \gamma$ (or $\pi^{0} \rightarrow \gamma$ in two dimensions) should vanish in the chiral phase, and be replaced by amplitudes involving the $\sigma$ meson. From a technical perspective, the common point of all these studies is the use at some stage of the imaginary time formalism, in the limit of vanishing external momenta. ${ }^{2}$

In another study, one of us [13] studied how the neutral

\footnotetext{
${ }^{1}$ This does not contradict the well established fact that the coefficient of the axial anomaly is temperature independent (see [3-7], for instance). Indeed, it has been shown in [8-10] that the relationship between the anomaly and the amplitude can be modified by the existence of an additional four-vector $U_{\mu}$ (the four-velocity of the plasma in the observer's frame) that can enter the general form of thermal amplitudes.

${ }^{2}$ There are two reasons why the small momentum limit is usually considered in this problem. The first one is that this limit provides an important technical simplification. The second reason is that this limit is appropriate if one wants to know the effective couplings between photons and mesons in the low energy regime, in order to write an effective Lagrangian for instance.
}

pion decay amplitude depends on the kinematical configuration of the external legs in order to explain the discrepancies found between $[1,2]$ and calculations performed in the real time formalism by [14-16]. To that purpose, the $\pi^{0} \gamma \gamma$ amplitude has been calculated at finite $T$ in the real time formalism, in the limit of small external momenta. It appeared that this limit cannot be uniquely defined (it depends on the path followed to reach the zero momenta point) and that the results of $[1,2,11,12]$ concerning this amplitude do not correspond to its on-shell value, but to a different way of reaching the limit. ${ }^{3}$ Additionally, the conclusion according to which the pion decay amplitude vanishes above the critical point appeared to be questionable since the physical (on-shell) amplitude has a nonvanishing limit at the critical point.

To accommodate this result with the general arguments provided in $[1,2,12]$, one can notice that both Pisarski's symmetry argument $[1,2]$ and Salcedo's argument [12] amount to the fact that one power of the quark mass (the mass the quarks acquire through the spontaneous breakdown of chiral symmetry, via their coupling to the average value of the $\sigma$ field) appears in the numerator when evaluating the Dirac trace associated to anomalous amplitudes. Therefore, since the average value $\langle\sigma\rangle$ goes to zero in the chiral phase, the numerator vanishes above the critical point. Implicit in the argument is the fact that the correct dimension is provided by inverse powers of the temperature (as opposed to powers of the quark mass), as is the case in the imaginary time formalism at the static point. In other words, this argument is valid only if the denominator does not vanish when the mass goes to zero. This is precisely what fails when the amplitude is calculated on shell. One gets as expected one power of $m$ $=g\langle\sigma\rangle$ in the numerator, but the denominator turns out to be $1 / m T$.

\footnotetext{
${ }^{3}$ The reason for this is easy to understand: since the energy variables are discrete in the imaginary time formalism, the only way one can consider the "zero momenta limit" in this formalism is to set first the discrete bosonic energies to zero, and then take the limit of zero three momenta. This way, the external momenta are forced to be spacelike.
} 
The remaining power of $m$ in the denominator indicates that the infrared or collinear behavior of the triangle diagram worsens when $m \rightarrow 0$. In fact, as noted in [13] and [17], the constituent quark mass $m$ ceases to be the relevant infrared regulator when $m$ is smaller than $g T$, and should be replaced by a thermal mass of order $g T$ that does not vanish in the chiral limit. Since an additional property of fermionic thermal masses is that they respect chiral symmetry, this thermal mass cannot appear in the Dirac trace. As a consequence, the result $\mathrm{m} / \mathrm{m} T$ obtained for the on-shell amplitude in the bare theory becomes $m / m_{\mathrm{th}} T$ after one has resummed the quark thermal mass $m_{\mathrm{th}} \sim g T$ (if $m \ll m_{\mathrm{th}}$ ). The consequence of this regularization is that the resummed on-shell decay amplitude vanishes in the chiral limit. In other words, Pisarski's symmetry arguments holds for the physical amplitude only after a proper regularization (in order to get rid of all potential infrared or collinear singularities) has been issued.

Essential in this discussion is the influence of the kinematical conditions for the external legs on the infrared behavior of a thermal amplitude, since it can dramatically alter one's conclusions. The second important point is that a calculation in the imaginary time formalism at the static point does not give a physical amplitude. Therefore, it would be interesting to test the second half of Pisarski's conclusions, related to the $\pi^{0} \sigma \rightarrow 2 \gamma$ amplitude, by calculating this amplitude in the real time formalism and studying how it depends on the kinematics (up to now, this amplitude has only been considered at the static point). Since this amplitude is given by a four-point function, it is a very complicated task to extract this behavior in its full generality. There is, however, a toy model in which this kind of study can be done quite simply: the two-dimensional linear $\sigma$ model. Indeed, in this model the neutral pion decays into a single photon, and that analogous to the four-point amplitude suggested by Pisarski would be $\pi^{0} \sigma \rightarrow \gamma$, which, being a three-point function, is rather easy to calculate.

The present paper is devoted to an analysis of the anomalous amplitudes in the two-dimensional $\sigma$ model at finite temperature and chemical potential. We consider both the pion decay $\pi^{0} \rightarrow \gamma$ two-point function and the $\pi^{0} \sigma \rightarrow \gamma$ three-point function. Emphasis is put on studying how these functions depend on the kinematics in the limit of small external momenta, near the chiral limit ( $m$ small compared to $\mu$ and $T$ ).

The structure of the paper is as follows. In Sec. II we define the model, as well as some notations and shorthands that will be used extensively later. In Sec. III, we calculate the amplitude for the $\pi^{0} \rightarrow \gamma$ decay, and reduce it to a very compact form. In Sec. IV, we study the amplitude of $\pi^{0} \sigma$ $\rightarrow \gamma$. Although a priori much more involved, this amplitude can also be reduced to a simple expression. All our results are expressed in terms of some function $I(K)$ defined by an integral. Basic properties and limits of this function are derived in Appendix A. Finally, in Appendix B we derive some relations between a few integrals that appear in intermediate stages of Sec. IV.

\section{CONVENTIONS AND NOTATIONS}

We consider the two-dimensional linear $\sigma$ model $[18,19]$ (more details on this model can be found in [20-22]) with

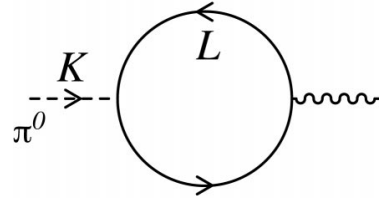

FIG. 1. One-loop $\pi^{0} \gamma$ amplitude.

two quark flavors, in which the mesons are coupled to quark fields as indicated by the following Lagrangian:

$$
\mathcal{L} \equiv i \bar{\Psi} \not D \Psi-2 g \bar{\Psi}\left(\sigma t_{0}+i \pi \cdot t \gamma^{5}\right) \Psi,
$$

where $t_{0}=1 / 2$ and $\operatorname{Tr}\left(t^{a} t^{b}\right)=\delta^{a b} / 2$. We recall that in two dimensions the Dirac algebra is defined by the following set of relations:

$$
\begin{aligned}
\left\{\gamma^{\mu}, \gamma^{\nu}\right\} & =2 g^{\mu \nu}, \\
\gamma^{5} & =\frac{1}{2} \epsilon^{\mu \nu} \gamma_{\mu} \gamma_{\nu},
\end{aligned}
$$

where $\epsilon^{\mu \nu}$ is the two-dimensional Levi-Civita tensor, normalized by $\epsilon^{01}=+1$. For later use, let us quote first a generic trace formula:

$$
\begin{aligned}
\operatorname{Tr}\left(\mathbb{A} \not B C C \gamma^{5} \gamma^{\mu}\right)= & A \cdot B \operatorname{Tr}\left(\mathbb{C} \gamma^{5} \gamma^{\mu}\right)-A \cdot C \operatorname{Tr}\left(\not B \gamma^{5} \gamma^{\mu}\right) \\
& +B \cdot C \operatorname{Tr}\left(\mathbb{A} \gamma^{5} \gamma^{\mu}\right) .
\end{aligned}
$$

In order to keep the following expressions compact, it is helpful to define the "dual" of a given vector by

$$
\widetilde{A}^{\mu} \equiv \epsilon^{\mu \nu} A_{\nu},
$$

as well as the "wedge product" of two vectors:

$$
A \wedge B \equiv \epsilon^{\mu \nu} A_{\mu} B_{\nu} .
$$

According to these definitions, we have the obvious relations

$$
\begin{aligned}
\widetilde{\widetilde{A}} & =A, \\
A \wedge B & =A \cdot \widetilde{B}, \\
(A \wedge B)^{2} & =(A \cdot B)^{2}-A^{2} B^{2} .
\end{aligned}
$$

When $A+B+C=0$, we have also

$$
A \wedge B=B \wedge C=C \wedge A .
$$

Finally, we have

$$
\operatorname{Tr}\left(\mathbb{A} \gamma^{5} \gamma^{\mu}\right)=-2 \widetilde{A}^{\mu}
$$

\section{III. $\pi^{0} \rightarrow \gamma$ AMPLITUDE}

\section{A. Retarded amplitude}

We consider first the one-loop contribution to the $\pi^{0}$ $\rightarrow \gamma$ decay amplitude depicted in Fig. 1. The Feynman rules 
for the retarded-advanced formalism ${ }^{4}[23-25]$ give for the retarded amplitude the following expression,

$$
\begin{aligned}
\Pi_{R}^{\mu}(K)= & -e g \int \frac{d^{2} L}{(2 \pi)^{2}} \operatorname{Tr}\left((\boldsymbol{L}+m) \gamma^{\mu}(\boldsymbol{L}+\mathbb{K}+m) \gamma^{5}\right) \\
& \times\left\{n_{F}\left(l_{0}, \mu\right) \operatorname{Disc} \Delta_{R}(L) \Delta_{R}(L+K)\right. \\
& \left.+n_{F}\left(l_{0}+k_{0}, \mu\right) \operatorname{Disc} \Delta_{R}(L+K) \Delta_{A}(L)\right\},
\end{aligned}
$$

where $\Delta_{R, A}(L) \equiv i /\left(L^{2}-m^{2} \pm i l_{0} \epsilon\right)$ are the retarded and advanced propagators, $n_{F}\left(l_{0}, \mu\right) \equiv 1 /\left[\exp \left(\left(l_{0}-\mu\right) / T\right)+1\right]$ is the Fermi-Dirac distribution function, and where the notation "Disc" denotes the discontinuity across the real energy axis:

$$
\operatorname{Disc} \Delta_{R}(L) \equiv \Delta_{R}(L)-\Delta_{A}(L)=2 \pi \epsilon\left(l_{0}\right) \delta\left(L^{2}-m^{2}\right) .
$$

The expression of the trace is very simple,

$$
\operatorname{Tr}\left((\boldsymbol{L}+m) \gamma^{\mu}(\boldsymbol{L}+\mathbb{K}+m) \boldsymbol{\gamma}^{5}\right)=-2 m \widetilde{K}^{\mu},
$$

and, in particular, it makes obvious the fact that $\Pi_{R}^{\mu}$ is transverse with respect to the photon momentum. Moreover, being independent of the loop momentum $L$, it can be immediately factorized out of the integral.

\section{B. Zero momentum limit}

At this point, it is convenient to perform the change of variable $L+K \rightarrow-L$ on the second term of Eq. (9) in order to make the expression more symmetric. Then, the Dirac distributions hidden in the discontinuities make the integration over $l_{0}$ trivial, which gives ${ }^{5}$

$$
\begin{aligned}
\Pi_{R}^{\mu}(K)= & -2 \operatorname{imeg} \widetilde{K}^{\mu} \int_{-\infty}^{+\infty} \frac{d l}{2 \pi} \frac{n_{F}\left(-\omega_{l}, \mu\right)-n_{F}\left(\omega_{l}, \mu\right)}{2 \omega_{l}} \\
& \times \sum_{\eta= \pm 1} \frac{1}{2 L_{\eta} \cdot K+K^{2}}
\end{aligned}
$$

where we denote $\omega_{l} \equiv \sqrt{l^{2}+m^{2}}$ and $L_{\eta} \equiv\left(\eta \omega_{l}, l\right)$. It is now trivial to perform an expansion in powers of the external momentum $K$. The first term in this expansion, of degree 0 in $K$, vanishes because the corresponding integrand is an odd function of $l$. The first nonvanishing term in this expansion comes at the next order, and is of degree 1 in $K$ :

\footnotetext{
${ }^{4}$ One should pay special attention to the chemical potential. Indeed, one should use a chemical potential $-\mu$ in statistical weights where the Feynman rules give an argument $-k_{0}$. In other words, $\mu$ appears in the formalism to account for the fact that the fermions carry some conserved charge, and the sign of this charge for a given propagator depends on how one orientates the propagator.

${ }^{5} \mathrm{We}$ have dropped the R/A prescription for the denominator, since it can easily be recovered at the very end of the calculation by substituting $k_{0} \rightarrow k_{0}+i 0^{+}$.
}

$$
\Pi_{R}^{\mu}(K)=\operatorname{imeg} \widetilde{K}^{\mu} I(K)
$$

where $I(K)$ is a homogeneous function of degree 0 in $K$, containing the nontrivial part of the momentum dependence and defined by

$$
I(K) \equiv \int_{-\infty}^{+\infty} \frac{d l}{2 \pi} \frac{n_{F}\left(-\omega_{l}, \mu\right)-n_{F}\left(\omega_{l}, \mu\right)}{2 \omega_{l}} \frac{K^{2}}{\left(L_{+} \cdot K\right)^{2}} .
$$

This integral is studied in some important limits in Appendix A.

\section{Discussion}

We observe for this amplitude the same features as in four dimensions. The most striking effect is related to what happens near the chiral limit $m \rightarrow 0$, and is visible in formulas (A7) and (A14). In the limit $m / T, m / \mu \rightarrow 0$, the function $I(K)$ behaves as follows:

If $K^{2}=0, \quad I(K)=\frac{1}{2 \pi m^{2}}$.

If $\quad K^{2} \neq 0 \quad$ and $k_{0} \neq 0, \quad I(K)=\frac{1}{8 m T} \frac{k_{0}}{\sqrt{K^{2}}} \frac{1}{\cosh ^{2}(\mu / 2 T)}$.

If $k_{0}=0, \quad I(K)= \begin{cases}\frac{7 \zeta(3)}{8 \pi^{3} T^{2}} \quad \text { if } \quad \mu \ll T, \\ -\frac{1}{4 \pi \mu^{2}} \quad \text { if } T \ll \mu .\end{cases}$

The configuration obtained with $k_{0}=0$ and $\mu \ll T$ corresponds to Salcedo's result, previously obtained in the imaginary time formalism at the static point. This point is particular because the first term in the expansion of $I(K)$ at small $m$ vanishes if $k_{0}=0$. For any other point, the expansion starts with a term behaving like $I(K) \sim 1 / m T$. However, as one turns the chemical potential on, we see that this leading term is exponentially suppressed when $\mu \gg T$. As a consequence, in a dense and cold system, the function $I(K)$ starts by a term in $1 / \mu^{2}$, whether $k_{0}=0$ or not.

The reason why the on-shell value is so singular when $m \rightarrow 0$ is related to collinear singularities: in $1+1$ dimensions, all the spatial vectors are aligned, so that we are always at the most singular point. ${ }^{6}$ Here also, in order to be able to apply Pisarski's argument, one should first regularize the theory by resumming a thermal mass $[13,17]$. Then, all

\footnotetext{
${ }^{6}$ This is to be contrasted with what happens in four dimensions: there the collinear singularities are softened by subsequent angular integrations, so that the on-shell amplitude is not exceptionally singular $[13,16]$.
} 
the powers of $m$ in the denominators would be replaced by powers of the thermal mass, leaving an uncompensated power of $m \rightarrow 0$ in the numerator. From Eq. (13), we can write an effective Lagrangian coupling the neutral pion to the photon:

$$
\begin{aligned}
\mathcal{L}_{\pi^{o} \gamma} & =-e g m \epsilon_{\mu \nu} \int d^{2} x A^{\mu}(x) I\left(i \partial_{x}\right) \partial_{x}^{\nu} \pi^{0}(x) \\
& =-e g^{2} \epsilon_{\mu \nu} \int d^{2} x A^{\mu}(x) I\left(i \partial_{x}\right)\langle\sigma\rangle \partial_{x}^{\nu} \pi^{0}(x) .
\end{aligned}
$$

This result completes the effective coupling found by Salcedo in [12], by incorporating all the nonlocal terms. The reason why the nonlocality of this coupling has been missed in [12] can of course be traced back in the choice of considering only amplitudes at the static point.

We can also make here a general comment regarding the fact that the symmetry argument implying that the $\pi^{0} \gamma$ amplitude vanishes in the chiral limit is not verified in the above one-loop calculation. In fact, this symmetry argument applies to the full amplitude, but not necessarily at the level of individual diagrams. More precisely, since the chiral limit corresponds to $m \rightarrow 0$, the argument will hold for any contribution that remains finite (in particular, IR finite) in this massless limit. The consequence of this problem is that this symmetry is manifest in perturbation theory only for sets of diagrams that are IR finite. This is why it may be necessary to perform the resummation of a thermal mass (i.e., consider larger sets of diagrams whose sum is infrared finite) in order to recover the result implied by chiral symmetry.

\section{IV. $\pi^{0} \sigma \rightarrow \gamma$ AMPLITUDE}

\section{A. Retarded amplitude}

We now consider the one-loop contribution to the $\pi^{0} \sigma$ $\rightarrow \gamma$ amplitude, represented in Fig. 2. In the retardedadvanced formalism, the $\Gamma_{A R R}^{\mu}$ component of the vertex receives the following contribution from the first diagram:

$$
\begin{aligned}
& \Gamma_{A R R}^{\mu,(1)}(K, P, S)=i e g^{2} \int \frac{d^{2} L}{(2 \pi)^{2}} \operatorname{Tr}\left((\boldsymbol{L}+m) \gamma^{5}(\boldsymbol{L}-\boldsymbol{P}+m)\right. \\
& \left.\times \gamma^{\mu}(\boldsymbol{L}+\$+m)\right)\left\{n_{F}\left(l_{0}, \mu\right) \operatorname{Disc} \Delta_{R}(L)\right. \\
& \times \Delta_{R}(L+S) \Delta_{A}(L-P)+n_{F}\left(l_{0}-p_{0}, \mu\right) \\
& \times \operatorname{Disc} \Delta_{R}(L-P) \Delta_{R}(L) \Delta_{R}(L+S) \\
& +n_{F}\left(l_{0}+s_{0}, \mu\right) \operatorname{Disc} \Delta_{R}(L+S) \\
& \left.\times \Delta_{A}(L) \Delta_{A}(L-P)\right\} \text {. }
\end{aligned}
$$

Again, the expression becomes simpler if we perform the changes of variables $L-P \rightarrow L$ on the second term, and $L$ $+S \rightarrow L$ on the third one. This enables one to have common statistical weight and discontinuity for the three terms, the tradeoff being that the trace becomes different for the three terms. We can apply the same manipulations to the contribu-

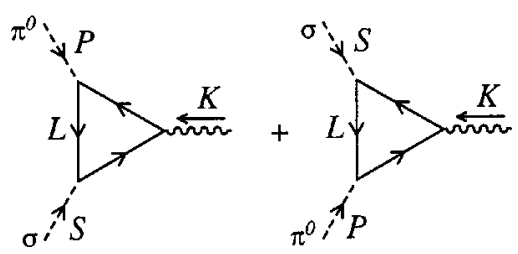

FIG. 2. One-loop $\pi^{0} \sigma \gamma$ amplitude.

tion of the second diagram. With the additional change $L$ $\rightarrow-L$ on the second diagram, we can merge the two contributions and find

$$
\begin{aligned}
\Gamma_{A R R}^{\mu}(K, P, S)= & \text { ieg } \int_{-\infty}^{+\infty} \frac{d l}{2 \pi} \frac{n_{F}\left(-\omega_{l}, \mu\right)-n_{F}\left(\omega_{l}, \mu\right)}{2 \omega_{l}} \\
& \times \sum_{\eta= \pm 1}\left\{\frac{\operatorname{Tr}_{a}^{\mu}}{\left(2 L_{\eta} \cdot K+K^{2}\right)\left(-2 L_{\eta} \cdot S+S^{2}\right)}\right. \\
& +\frac{\operatorname{Tr}_{b}^{\mu}}{\left(2 L_{\eta} \cdot P+P^{2}\right)\left(-2 L_{\eta} \cdot K+K^{2}\right)} \\
& \left.+\frac{\operatorname{Tr}_{c}^{\mu}}{\left(2 L_{\eta} \cdot S+S^{2}\right)\left(-2 L_{\eta} \cdot P+P^{2}\right)}\right\}
\end{aligned}
$$

where the traces are given by

$$
\begin{aligned}
& \operatorname{Tr}_{a}^{\mu}=-2 m^{2} \operatorname{Tr}\left(\boldsymbol{P} \gamma^{5} \gamma^{\mu}\right)-2 L_{\eta} \cdot S \operatorname{Tr}\left(\boldsymbol{L}_{\eta} \gamma^{5} \gamma^{\mu}\right) \\
& -\operatorname{Tr}\left(L_{\eta} \$ \mathbb{K} \gamma^{5} \gamma^{\mu}\right) \\
& \operatorname{Tr}_{b}^{\mu}=-2 m^{2} \operatorname{Tr}\left(\boldsymbol{P} \gamma^{5} \gamma^{\mu}\right)+2 L_{\eta} \cdot P \operatorname{Tr}\left(\boldsymbol{L}_{\eta} \gamma^{5} \gamma^{\mu}\right) \\
& -\operatorname{Tr}\left(\mathbb{K} \boldsymbol{P} \boldsymbol{L}_{\eta} \gamma^{5} \gamma^{\mu}\right) \text {, } \\
& \operatorname{Tr}_{a}^{\mu}=-2 m^{2} \operatorname{Tr}\left(\boldsymbol{P} \gamma^{5} \gamma^{\mu}\right)-\operatorname{Tr}\left(\$ \boldsymbol{L}_{\eta} \not \boldsymbol{P} \gamma^{5} \gamma^{\mu}\right)
\end{aligned}
$$

\section{B. Zero momenta limit}

We can now proceed with the expansion in powers of the external momenta. The first term in this expansion is of degree -1 in the external momenta. An explicit calculation of this term shows that it vanishes thanks to energy-momentum conservation: $P+K+S=0$. The next term, of degree 0 in the external momenta, vanishes also because the corresponding integrand is an odd function of $l$. Therefore, the first nonvanishing term is of degree 1 in the external momenta. An explicit extraction of this term gives 


$$
\begin{aligned}
\Gamma_{A R R}^{\mu}(K, P, S)= & i \frac{e g^{2}}{8} \int_{-\infty}^{+\infty} \frac{d l}{2 \pi} \frac{n_{F}\left(-\omega_{l}, \mu\right)-n_{F}\left(\omega_{l}, \mu\right)}{2 \omega_{l}} \sum_{\eta= \pm 1}\left\{-m^{2} \operatorname{Tr}\left(\boldsymbol{P} \gamma^{5} \gamma^{\mu}\right)\left[\frac{P^{2}}{\left(L_{\eta} \cdot P\right)^{2}} \frac{S^{2}}{\left(L_{\eta} \cdot S\right)^{2}}+\frac{S^{2}}{\left(L_{\eta} \cdot S\right)^{2}} \frac{K^{2}}{\left(L_{\eta} \cdot K\right)^{2}}\right.\right. \\
& \left.+\frac{K^{2}}{\left(L_{\eta} \cdot K\right)^{2}} \frac{P^{2}}{\left(L_{\eta} \cdot P\right)^{2}}+\frac{1}{\left(L_{\eta} \cdot K\right)\left(L_{\eta} \cdot P\right)\left(L_{\eta} \cdot S\right)}\left(\frac{K^{4}}{L_{\eta} \cdot K}+\frac{P^{4}}{L_{\eta} \cdot P}+\frac{S^{4}}{L_{\eta} \cdot S}\right)\right] \\
& +\frac{\operatorname{Tr}\left(\boldsymbol{L}_{\eta} \gamma^{5} \gamma^{\mu}\right)}{L_{\eta} \cdot K}\left[\frac{K^{2}}{L_{\eta} \cdot K}\left(\frac{P^{2}}{L_{\eta} \cdot P}-\frac{S^{2}}{L_{\eta} \cdot S}\right)+\frac{S^{4}}{\left(L_{\eta} \cdot S\right)^{2}}-\frac{P^{4}}{\left(L_{\eta} \cdot P\right)^{2}}\right]+\frac{\operatorname{Tr}\left(\boldsymbol{L}_{\eta} \$ K \gamma^{5} \gamma^{\mu}\right)}{\left(L_{\eta} \cdot K\right)\left(L_{\eta} \cdot S\right)}\left[\frac{S^{2}}{L_{\eta} \cdot S}-\frac{K^{2}}{L_{\eta} \cdot K}\right] \\
& \left.+\frac{\operatorname{Tr}\left(\boldsymbol{K} \boldsymbol{P} \boldsymbol{L}_{\eta} \gamma^{5} \gamma^{\mu}\right)}{\left(L_{\eta} \cdot P\right)\left(L_{\eta} \cdot K\right)}\left[\frac{K^{2}}{L_{\eta} \cdot K}-\frac{P^{2}}{L_{\eta} \cdot P}\right]+\frac{\operatorname{Tr}\left(\$ \boldsymbol{L}_{\eta} \boldsymbol{P} \gamma^{5} \gamma^{\mu}\right)}{\left(L_{\eta} \cdot S\right)\left(L_{\eta} \cdot P\right)}\left[\frac{P^{2}}{L_{\eta} \cdot P}-\frac{S^{2}}{L_{\eta} \cdot S}\right]\right\} .
\end{aligned}
$$

At this point, we can make use of Eqs. (3) and (8). It is now obvious that the result can be expressed in terms of the following integrals ${ }^{7}$

$$
\begin{aligned}
J_{A B} \equiv & \int_{-\infty}^{+\infty} \frac{d l}{2 \pi} \frac{n_{F}\left(-\omega_{l}, \mu\right)-n_{F}\left(\omega_{l}, \mu\right)}{2 \omega_{l}} \frac{1}{L_{+} \cdot A} \frac{1}{L_{+} \cdot B} \\
J_{A A} \equiv & \int_{-\infty}^{+\infty} \frac{d l}{2 \pi} \frac{n_{F}\left(-\omega_{l}, \mu\right)-n_{F}\left(\omega_{l}, \mu\right)}{2 \omega_{l}} \frac{1}{\left(L_{+} \cdot A\right)^{2}} \\
= & \frac{I(A)}{A^{2}} \\
J_{A A B}^{\mu} \equiv & \int_{-\infty}^{+\infty} \frac{d l}{2 \pi} \frac{n_{F}\left(-\omega_{l}, \mu\right)-n_{F}\left(\omega_{l}, \mu\right)}{2 \omega_{l}} \\
& \times \frac{L_{+}^{\mu}}{\left(L_{+} \cdot A\right)^{2}} \frac{1}{L_{+} \cdot B}, \\
J_{A A B C} \equiv & m^{2} \int_{-\infty}^{+\infty} \frac{d l}{2 \pi} \frac{n_{F}\left(-\omega_{l}, \mu\right)-n_{F}\left(\omega_{l}, \mu\right)}{2 \omega_{l}} \\
& \times \frac{1}{\left(L_{+} \cdot A\right)^{2}} \frac{1}{L_{+} \cdot B} \frac{1}{L_{+} \cdot C}, \\
J_{A A B B} \equiv & m^{2} \int_{-\infty}^{+\infty} \frac{d l}{2 \pi} \frac{n_{F}\left(-\omega_{l}, \mu\right)-n_{F}\left(\omega_{l}, \mu\right)}{2 \omega_{l}} \\
& \times \frac{1}{\left(L_{+} \cdot A\right)^{2}} \frac{1}{\left(L_{+} \cdot B\right)^{2}},
\end{aligned}
$$

where $A, B, C$ can be any of $P, S, K$.

\section{Transversality}

The major difference compared to the case of the $\pi^{0} \gamma$ amplitude is that the above three-point amplitude is not manifestly transverse with respect to the photon momentum.

\footnotetext{
${ }^{7}$ Note that we need only $J_{A A B C}$ when $A+B+C=0$. Having calculated $J_{A A B C}$ under this restrictive assumption, one cannot obtain $J_{A A B B}$ from it by enforcing $C=B$.
}

The fact that the Dirac's traces depend upon the loop momentum $L$ indicates that it could be necessary to calculate all the integrals before one can see the transversality. The situation is not so intricate though, since it happens that we only need to establish some relations between the various integrals defined in Eqs. (21). In Appendix B, we show how the last three integrals of Eq. (21) can be expressed as functions of the first two. Using the relations given in Eqs. (B2), (B5), and (B6), as well as Eq. (B7), it is a simple matter of algebra to check the transversality of the $\pi^{0} \sigma \gamma$ amplitude with respect to $K$, without the need of calculating the various $J_{A A}$ and $J_{A B}$.

Once we know that the result can indeed be written as $\Gamma^{\mu}=\Gamma \widetilde{K}^{\mu}$, one can contract the amplitude with $S$, for instance, in order to extract the coefficient $\Gamma$. A straightforward calculation gives

$$
\Gamma_{A R R}^{\mu}(K, P, S)=i \operatorname{eg}^{2} \widetilde{K}^{\mu} F(P, S),
$$

with

$$
F(P, S) \equiv \frac{P^{2} S^{2} I(S)+(P \cdot S)\left[P^{2} I(P)+(P \cdot K) I(K)\right]}{(P \wedge S)^{2}},
$$

where implicitly $K=-P-S$. At first sight, this expression could become infinite whenever two momenta become parallel. However, one can check that this is not the case, because the numerator behaves like $(P \wedge S)^{2}$ when $P \wedge S$ becomes small.

\section{Discussion}

We see that this three-point amplitude involving the $\sigma$ field depends on the same function $I($.$) defined above, and$ has one power of the mass $m$ less when compared to the $\pi^{o} \gamma$ amplitude, in agreement with the general arguments of $[1,2,12]$.

Again, it is found that this limit depends on the kinematics, i.e., on the way one is approaching the zero momenta limit. In particular, the way this amplitude depends on $m$ at small $m$ depends strongly on the kinematics. This is to be 
contrasted with the result of [12], which only picked one particular limit. Except at the static point $\left(k_{0}=p_{0}=s_{0}=0\right)$, this amplitude becomes singular at the critical point where $m \rightarrow 0$, indicating the necessity of regularizing the fermion propagator by a thermal mass. After this resummation has been performed, this amplitude is regular but does not vanish in the chirally symmetric phase. Therefore, the conjecture of [2] holds, but only after infrared regularization.

One can also write an effective coupling associated with this amplitude:

$$
\begin{aligned}
\mathcal{L}_{\pi^{0} \sigma \gamma}= & e g^{2} \epsilon_{\mu \nu} \int d^{2} x A^{\mu}(x) F\left(i \partial_{x_{1}}, i \partial_{x_{2}}\right) \\
& \times\left.\left[\sigma\left(x_{2}\right) \partial_{x_{1}}^{\nu} \pi^{0}\left(x_{1}\right)+\pi^{0}\left(x_{1}\right) \partial_{x_{2}}^{\nu} \sigma\left(x_{2}\right)\right]\right|_{x_{1}=x_{2}=x} .
\end{aligned}
$$

Of course, if one uses Eq. (A1), one finds a local limit for this effective coupling in the limit of zero temperature and density.

\section{CONCLUSIONS}

In this paper, we have studied the $\pi^{0} \gamma$ and $\pi^{0} \sigma \gamma$ amplitudes in the two-dimensional $\sigma$ model at finite temperature and density. For both of these amplitudes, the zero momenta limit is not unique and strongly depends on the kinematical configuration. In particular, the imaginary time formalism should be used with great care when looking at this limit. Indeed, if one first sets the external discrete energies to zero, then all the information regarding the nonlocality of the amplitude is lost, and in particular the physical limit cannot be recovered. A proper way to use the imaginary time formalism would be to perform the sum over the loop discrete energies while keeping nonzero external discrete energies. After that, one should perform the analytical continuation to real external energies, and only then consider the zero momenta limit.

The other conclusion of this work is that collinear or infrared singularities spoil the general symmetry arguments given in $[1,2,12]$ to justify the nullity of the pion decay into photons in the chirally symmetric phase: the on-shell decay amplitude in the bare theory does not vanish. For these arguments to be valid, one should perform the resummation of a thermal mass that will regularize the fermion propagators.

If such a regularization is used, then the conclusion is that $\pi^{0} \rightarrow \gamma$ vanishes when $m \rightarrow 0$, while $\pi^{0} \sigma \rightarrow \gamma$ does not, in agreement with the conjecture of [1].

\section{ACKNOWLEDGMENTS}

We thank the Erwin Schrödinger Institute for support and hospitality during the worshop "BRST cohomology, quantization and anomalies," where this work started. The work of
F.G. is supported by DOE under Grant No. DE-AC0298CH10886.

\section{APPENDIX A: PROPERTIES OF THE FUNCTION $I(K)$}

\section{Vacuum limit}

The purpose of this appendix is to study the integral $I(K)$ since all the quantities calculated in this paper can be expressed in terms of this function. A first check is to look at the zero temperature and chemical potential limit of this function, which gives immediately

$$
\lim _{T, \mu \rightarrow 0^{+}} I(K)=\frac{1}{2 \pi m^{2}} .
$$

As one could expect, this $T=\mu=0$ limit does not exhibit any nonlocality in its momentum dependence, since this is a purely thermal feature.

\section{Transformation into a sum}

A convenient way to look at the high temperature or density limit is to turn the integral defining $I(K)$ in Eq. (14) into a sum, by making use of the following identity: ${ }^{8}$

$$
\frac{1}{e^{x}+1}=\frac{1}{2}-2 x \sum_{n=0}^{+\infty} \frac{1}{x^{2}+(2 n+1)^{2} \pi^{2}} .
$$

This identity enables one to rewrite $n_{F}\left(-\omega_{l}, \mu\right)$ $-n_{F}\left(\omega_{l}, \mu\right)$ as a series, from which it is straightforward to first obtain

$$
\begin{aligned}
I(K)= & -\frac{1}{\pi T^{2}} \frac{\kappa^{2}+1}{\kappa^{2}-1} \sum_{n=0}^{+\infty} \int_{-\infty}^{+\infty} d v \frac{v^{2}+\xi_{+}^{2}}{\left(v^{2}+\xi_{-}^{2}\right)^{2}} \\
& \times \frac{v^{2}+A_{n}^{2}}{\left(v^{2}+A_{n}^{2}\right)^{2}+B_{n}^{2}},
\end{aligned}
$$

where we denote

$$
\begin{aligned}
v & \equiv \frac{l}{T}, \quad \kappa \equiv k_{0} / k, \quad \xi_{ \pm}^{2} \equiv \frac{m^{2}}{T^{2}} \frac{\kappa^{2}}{\kappa^{2} \pm 1} \\
A_{n}^{2} & \equiv \pi^{2}(2 n+1)^{2}+\frac{m^{2}}{T^{2}}-\frac{\mu^{2}}{T^{2}}, \quad B_{n}^{2} \equiv 4 \pi^{2}(2 n+1)^{2} \frac{\mu^{2}}{T^{2}} .
\end{aligned}
$$

At this stage, it remains to perform term by term the integration over $d v$, which is elementary and yields

\footnotetext{
${ }^{8}$ To derive this formula, one can start from Mittag-Leffler's expansion of the cot function $[26,27]$ :

$$
\cot (z)=\frac{1}{z}+2 \sum_{n=1}^{+\infty} \frac{z}{z^{2}-n^{2} \pi^{2}} .
$$




$$
\begin{aligned}
\int_{-\infty}^{+\infty} d v & \frac{v^{2}+\xi_{+}^{2}}{\left(v^{2}+\xi_{-}^{2}\right)^{2}} \frac{v^{2}+A_{n}^{2}}{\left(v^{2}+A_{n}^{2}\right)^{2}+B_{n}^{2}} \\
= & -\frac{\pi}{2 \xi_{-}} \frac{\partial}{\partial \xi_{-}}\left[\frac{1}{\xi_{-}} \frac{\left(\xi_{+}^{2}-\xi_{-}^{2}\right)\left(A_{n}^{2}-\xi_{-}^{2}\right)}{\left(A_{n}^{2}-\xi_{-}^{2}\right)^{2}+B_{n}^{2}}\right. \\
& +\frac{A_{n}^{4}+B_{n}^{2}-2 \xi_{+}^{2} A_{n}^{2}+\left(\xi_{+}^{2}-\xi_{-}^{2}\right) \sqrt{A_{n}^{4}+B_{n}^{2}}+\xi_{+}^{2} \xi_{-}^{2}}{\left(A_{n}^{2}-\xi_{-}^{2}\right)^{2}+B_{n}^{2}} \\
& \times \sqrt{\left.\frac{\sqrt{A_{n}^{4}+B_{n}^{2}}+A_{n}^{2}}{2\left(A_{n}^{4}+B_{n}^{2}\right)}\right] .}
\end{aligned}
$$

\section{Chiral limit far from the light cone}

Another interesting limit is the chiral limit, where $m / T$ goes to zero, while $\mu / T$ is kept fixed. It is very easy to extract from the above formula a systematic expansion in powers of $m / T$. It is just a matter of expanding at small $\xi_{ \pm}$ the above expression, which gives for the first two orders

$$
I(K)=\frac{1}{m T}\left[\frac{k_{0}}{\sqrt{K^{2}}} F_{0}\left(\frac{\mu}{T}\right)+\frac{m}{T} \frac{K^{2}+2 k^{2}}{K^{2}} F_{1}\left(\frac{\mu}{T}\right)+\mathcal{O}\left(\frac{m^{2}}{T^{2}}\right)\right],
$$

where the coefficients are given by

$$
\begin{aligned}
& F_{0}\left(\frac{\mu}{T}\right)=\frac{1}{\pi^{2}} \operatorname{Re} \sum_{n=0}^{+\infty} \frac{1}{(2 n+1+i \mu / \pi T)^{2}}, \\
& F_{1}\left(\frac{\mu}{T}\right)=\frac{1}{\pi^{3}} \sum_{n=0}^{+\infty}(2 n+1) \frac{(2 n+1)^{2}-3 \mu^{2} / \pi^{2} T^{2}}{\left((2 n+1)^{2}+\mu^{2} / \pi^{2} T^{2}\right)^{3}} .
\end{aligned}
$$

Introducing the "digamma" function,

$$
\psi(z) \equiv \frac{d}{d z} \ln \Gamma(z),
$$

and a series representation of its first derivative,

$$
\psi^{\prime}\left(\frac{1+z}{2}\right)=4 \sum_{n=0}^{+\infty} \frac{1}{(2 n+1+z)^{2}},
$$

it is immediate to check ${ }^{9}$

\footnotetext{
${ }^{9}$ The equality on the first line is exact and comes from the formula $[26,27]$$$
\Gamma(z) \Gamma(1-z)=\frac{\pi}{\sin (\pi z)},
$$

while the limit $\mu \gg T$ in the last line is obtained from Stirling's expansion $[26,27]$ for $\Gamma(z)$.
}

$$
\begin{aligned}
F_{0}\left(\frac{\mu}{T}\right) & =\frac{1}{8 \pi^{2}}\left[\psi^{\prime}\left(\frac{1}{2}+i \frac{\mu}{2 \pi T}\right)+\psi^{\prime}\left(\frac{1}{2}-i \frac{\mu}{2 \pi T}\right)\right] \\
& =\frac{1}{8 \cosh ^{2}(\mu / 2 T)}, \\
F_{1}\left(\frac{\mu}{T}\right) & =-\frac{1}{32 \pi^{3}}\left[\psi^{\prime \prime}\left(\frac{1}{2}+i \frac{\mu}{2 \pi T}\right)+\psi^{\prime \prime}\left(\frac{1}{2}-i \frac{\mu}{2 \pi T}\right)\right] \\
& \approx\left\{\begin{array}{l}
\frac{7 \zeta(3)}{8 \pi^{3}} \quad \text { if } \mu \ll T \\
-\frac{T^{2}}{4 \pi \mu^{2}} \quad \text { if } T \ll \mu
\end{array}\right.
\end{aligned}
$$

\section{Light cone limit}

Note, however, that this expansion is not valid near the light cone. Indeed, its derivation assumed that a small $\mathrm{m} / \mathrm{T}$ would imply a small $\xi_{-}$, which is not true if $K^{2}$ is small (or equivalently $\kappa^{2} \approx 1$ ). Sufficiently close to the light cone, $\xi_{-}$ becomes large and a different kind of expansion must be considered. In this region of phase space, one can write

$$
\begin{aligned}
\lim _{K^{2} \rightarrow 0} I(K) & =\lim _{\xi_{-} \rightarrow+\infty} \frac{1}{2 \xi_{-} T^{2}} \frac{2}{\kappa^{2}-1} \sum_{n=0}^{+\infty} \frac{1}{\left[\xi_{-}+(2 n+1) \pi\right]^{2}} \\
& =\lim _{\xi_{-} \rightarrow+\infty} \frac{\xi_{-}}{m^{2}} \frac{1}{4 \pi^{2}} \psi^{\prime}\left(\frac{1}{2}+\frac{\xi_{-}}{2 \pi}\right),
\end{aligned}
$$

where we have used $\left(\kappa^{2}-1\right)^{-1} \approx \xi_{-}^{2} T^{2} / m^{2}$. Making use of Stirling's formula [26,27], we obtain

$$
\lim _{K^{2} \rightarrow 0} I(K)=\frac{1}{2 \pi m^{2}}
$$

We notice that the on-shell value of $I(K)$ is totally immune to corrections due to temperature or density. The origin of this property can be understood from Eq. (14): when $K^{2}$ $=0$, the denominator behaves like $k^{2} m^{4} / l^{4}$ (this is reminiscent of a collinear singularity cured by the mass $m$ ), and the integral is completely dominated by its ultraviolet sector. As a consequence, $I(K)$ is saturated by the vacuum contribution for this value of $K^{2}$. Away from the light cone, thermal corrections of order $1 / m T$ appear in $I(K)$.

\section{APPENDIX B: RELATIONS BETWEEN SOME INTEGRALS}

In this appendix, we establish some useful relations between the five integrals defined in Eq. (21). Let us start by the study of $J_{A A B}^{\mu}$ : this integral satisfies the $2 \times 2$ linear system

$$
\begin{aligned}
& A_{\mu} J_{A A B}^{\mu}=J_{A B}, \\
& B_{\mu} J_{A A B}^{\mu}=J_{A A},
\end{aligned}
$$

the resolution of which gives the two components of $J_{A A B}^{\mu}$ as functions of $J_{A A}$ and $J_{A B}$ : 


$$
J_{A A B}^{\mu}=\frac{\widetilde{B}^{\mu} J_{A B}-\widetilde{A}^{\mu} J_{A A}}{A \wedge B} .
$$

In order to obtain $J_{A A B B}$ it is convenient to define first the second rank tensor

$$
\begin{aligned}
J_{A A B B}^{\mu \nu} \equiv & \int_{-\infty}^{+\infty} \frac{d l}{2 \pi} \frac{n_{F}\left(-\omega_{l}, \mu\right)-n_{F}\left(\omega_{l}, \mu\right)}{2 \omega_{l}} \\
& \times \frac{L_{+}^{\mu}}{\left(L_{+} \cdot A\right)^{2}} \frac{L_{+}^{\nu}}{\left(L_{+} \cdot B\right)^{2}},
\end{aligned}
$$

from which we can obtain $J_{A A B B}$ as $g_{\mu \nu} J_{A A B B}^{\mu \nu}$. The three independent components of this symmetric tensor can be obtained via the resolution of the following $3 \times 3$ linear system

$$
\begin{aligned}
& A_{\mu} B_{\nu} J_{A A B B}^{\mu \nu}=J_{A B}, \\
& A_{\mu} A_{\nu} J_{A A B B}^{\mu \nu}=J_{B B},
\end{aligned}
$$

$$
B_{\mu} B_{\nu} J_{A A B B}^{\mu \nu}=J_{A A},
$$

which finally gives

$$
J_{A A B B}=\frac{2(A \cdot B) J_{A B}-A^{2} J_{A A}-B^{2} J_{B B}}{(A \wedge B)^{2}} .
$$

The same method can be applied to $J_{A A B C}$, which gives

$$
J_{A A B C}=\frac{A^{2} J_{A A}+B^{2} J_{A B}+C^{2} J_{A C}}{(A \wedge B)^{2}},
$$

under the assumption that $A+B+C=0$. We also need the following relation,

$$
J_{A B}+J_{B C}+J_{C A}=0
$$

which is valid when $A+B+C=0$.
[1] R. D. Pisarski, in From Thermal Field Theory to Neural Networks: A Day to Remember Tanguy Altherr, edited by P. Aurenche, P. Sorba, and G. Veneziano (World Scientific, Singapore, 1996).

[2] R. D. Pisarski, Phys. Rev. Lett. 76, 3084 (1996).

[3] H. Itoyama and A. H. Mueller, Nucl. Phys. B218, 349 (1983).

[4] L. Dolan and R. Jackiw, Phys. Rev. D 9, 3320 (1974).

[5] R. Baier and E. Pilon, Z. Phys. C 52, 339 (1991).

[6] A. Gomez Nicola and A. F. Alvarez-Estrada, Int. J. Mod. Phys. A 9, 1423 (1994).

[7] A. V. Smilga, Phys. Rev. D 45, 1378 (1992).

[8] R. D. Pisarski and M. G. H. Tytgat, Phys. Rev. D 54, 2989 (1996).

[9] R. D. Pisarski and M. H. G. Tytgat, Phys. Rev. Lett. 78, 3622 (1997).

[10] R. D. Pisarski and T. L. Trueman, and M. H. G. Tytgat, Phys. Rev. D 56, 7077 (1997).

[11] R. Baier, M. Dirks, and O. Kober, Phys. Rev. D 54, 2222 (1996).

[12] L. L. Salcedo, Phys. Rev. D 58, 125007 (1998).

[13] F. Gelis, Phys. Rev. D 59, 076004 (1999).

[14] C. Contreras and M. Loewe, Z. Phys. C 40, 253 (1988).
[15] A. Gomez Nicola and A. F. Alvarez-Estrada, Z. Phys. C 60, 711 (1993).

[16] S. Gupta and S. N. Nayak, TIFR/TH/97-03, hep-ph/9702205.

[17] S. P. Kumar, D. Boyanovsky, H. de Vega, and R. Holman, Phys. Rev. D 61, 065002 (2000).

[18] V. Koch, Int. J. Mod. Phys. E 6, 203 (1997).

[19] A. Bochkarev and J. I. Kapusta, Phys. Rev. D 54, 4066 (1996).

[20] S. Coleman, Ann. Phys. (N.Y.) 101, 239 (1976).

[21] S. Coleman, R. Jackiw, and L. Susskind, Ann. Phys. (N.Y.) 93, 267 (1975).

[22] M. E. Peskin and D. V. Schroeder, An Introduction to Quantum Field Theory (Addison-Wesley, New York, 1995), pp. 651-658.

[23] P. Aurenche and T. Becherrawy, Nucl. Phys. B379, 259 (1992).

[24] M. A. van Eijck and Ch. G. van Weert, Phys. Lett. B 278, 305 (1992).

[25] M. A. van Eijck, R. Kobes, and Ch. G. van Weert, Phys. Rev. D 50, 4097 (1994).

[26] B. Chabat, Analyse Complexe, Tome I (MIR, Moscow, 1990).

[27] S. Lang, Complex Analysis (Springer-Verlag, Berlin, 1993). 